\title{
Training in Bariatric and Metabolic Endoscopic Therapies
}

\author{
Pichamol Jirapinyo ${ }^{1,2}$ and Christopher C. Thompson ${ }^{1,2}$ \\ ${ }^{1}$ Division of Gastroenterology, Brigham and Women's Hospital, Boston, MA, ${ }^{2}$ Harvard Medical School, Boston, MA, USA
}

Bariatric endoscopy is an emerging subspecialty for gastroenterologists encompassing a broad array of procedures including primary endoscopic bariatric and metabolic therapies and the treatment of complications of bariatric surgery. In addition, comprehensive understanding of lifestyle intervention and pharmacotherapy are essential to successful outcomes. This review summarizes goals and steps of training for this emerging field. Clin Endosc 2018;51:430-438

Key Words: Endoscopic bariatric and metabolic therapies; EBMT; Bariatric; Metabolic; Training

\section{INTRODUCTION}

Obesity is a worsening pandemic with a worldwide prevalence that has nearly tripled since 1975. In 2016, more than 650 million adults worldwide had obesity and over 1.9 billion were overweight. This represented $13 \%$ and $39 \%$ of the world's adult population, respectively. ${ }^{1}$ Management of obesity involves lifestyle therapy pharmacotherapy and surgery. ${ }^{2}$ Despite being minimally invasive, comprehensive lifestyle intervention and pharmacotherapy are associated with approximately $5 \%$ total weight loss (\%TWL) and 5\% to 10\% TWL, respectively., ${ }^{3,4}$ In contradistinction, surgical intervention is effective and consistently induces greater than $25 \%$ TWL. ${ }^{5}$ Nevertheless, less than $1 \%$ of eligible patients elect to undergo surgery. ${ }^{6}$ Endoscopic bariatric and metabolic therapies (EBMTs) have recently been developed as an additional option for the treatment of obesity and its related comorbidities with relatively higher efficacy than lifestyle intervention and pharmacotherapy and potentially fewer adverse events than surgery.

Received: July 1, 2018 Accepted: September 18, 2018

Correspondence: Christopher C. Thompson

Division of Gastroenterology, Brigham and Women's Hospital, 75 Francis Street, Boston, MA 02115, USA

Tel: +1-617-525-8266, Fax: +1-617-264-6342, E-mail: cthompson@hms.harvard. edu

ORCID: https://orcid.org/0000-0002-6105-5270

cc This is an Open Access article distributed under the terms of the Creative Commons Attribution Non-Commercial License (http://creativecommons.org/ licenses/by-nc/3.0) which permits unrestricted non-commercial use, distribution, and reproduction in any medium, provided the original work is properly cited.
EBMTs may be classified in several ways. First, it may be arranged by anatomic location of the device or therapy being stomach versus small bowel. When the primary purpose of the procedure is considered, EBMTs may be classified as primary therapy, bridge therapy, revisional procedure and management of other complications. Based on the complexity of the procedures, EBMTs may also be categorized into level I procedures (less complex procedures that may be trained during the standard gastroenterology [GI] fellowship) and level II procedures (more complex procedures that require more advanced endoscopic training).

There are currently six Food and Drug Administration (FDA)-approved primary endoscopic therapies, with several other devices available worldwide. There are three FDA-approved intragastric balloons (IGBs), including a single fluid-filled balloon, a single gas-filled balloon, and a double fluid-filled balloon (Fig. 1A-C). ${ }^{7-12}$ The Overstitch Endoscopic Suturing System (Fig. 2) and the Incisionless Operating Platform (IOP) (Fig. 3) are approved for general tissue apposition. These devices are most commonly used to remodel the stomach in the treatment of obesity and weight regain (Fig. 1D, E). ${ }^{13-19}$ These procedures include Endoscopic Sutured Gastroplasty (ESG) and Primary Obesity Surgery Endoluminal (POSE) for primary obesity and Transoral Outlet Reduction (TORe) and Restorative Obesity Surgery Endoluminal (ROSE) for revisional work. Aspiration Therapy is similar to a percutaneous gastrostomy tube with a long internal fenestrated tube that allows removal of approximately $30 \%$ of ingested calories 


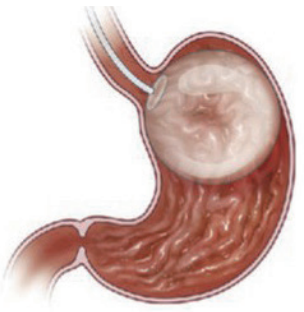

(A)

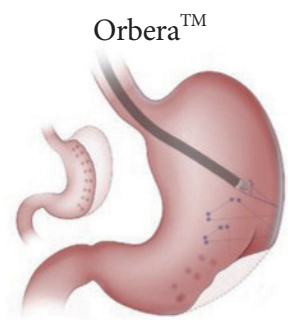

Endoscopic sutured

(D) gastroplasty

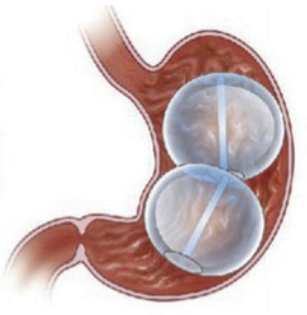

(B)

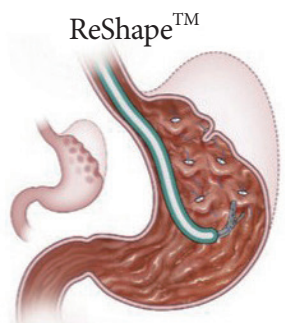

Primary obesity

(E)

surgery endoluminal

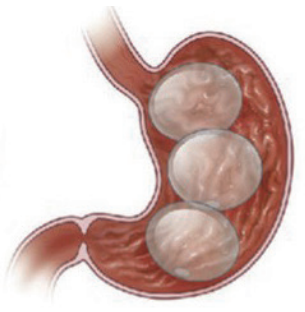

(C) Obalon ${ }^{\mathrm{TM}}$

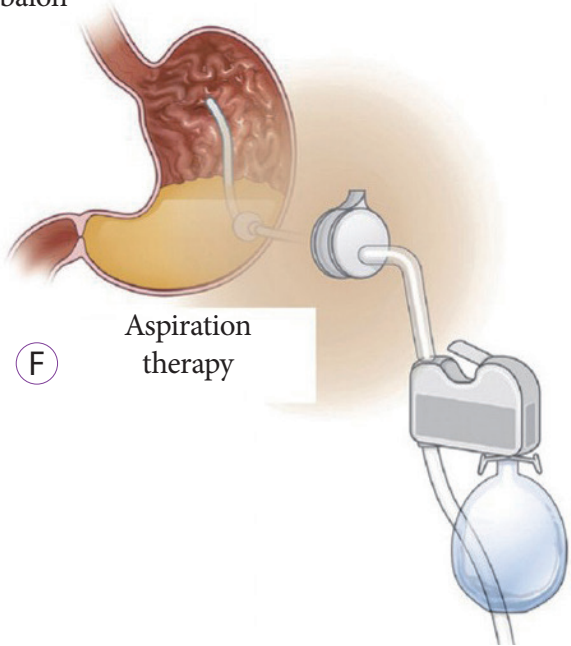

Fig. 1. Primary endoscopic bariatric and metabolic therapies. (A) Orbera ${ }^{\mathrm{TM}}$ Balloon. (B) ReShape ${ }^{\mathrm{TM}}$ Balloon. (C) Obalon ${ }^{\mathrm{TM}}$ Balloon. (D) Endoscopic Sutured Gastroplasty. (E) Primary Obesity Surgery Endoluminal. (F) Aspiration Therapy. Adapted from Jirapinyo et al., with permission from Elsevier. ${ }^{21}$
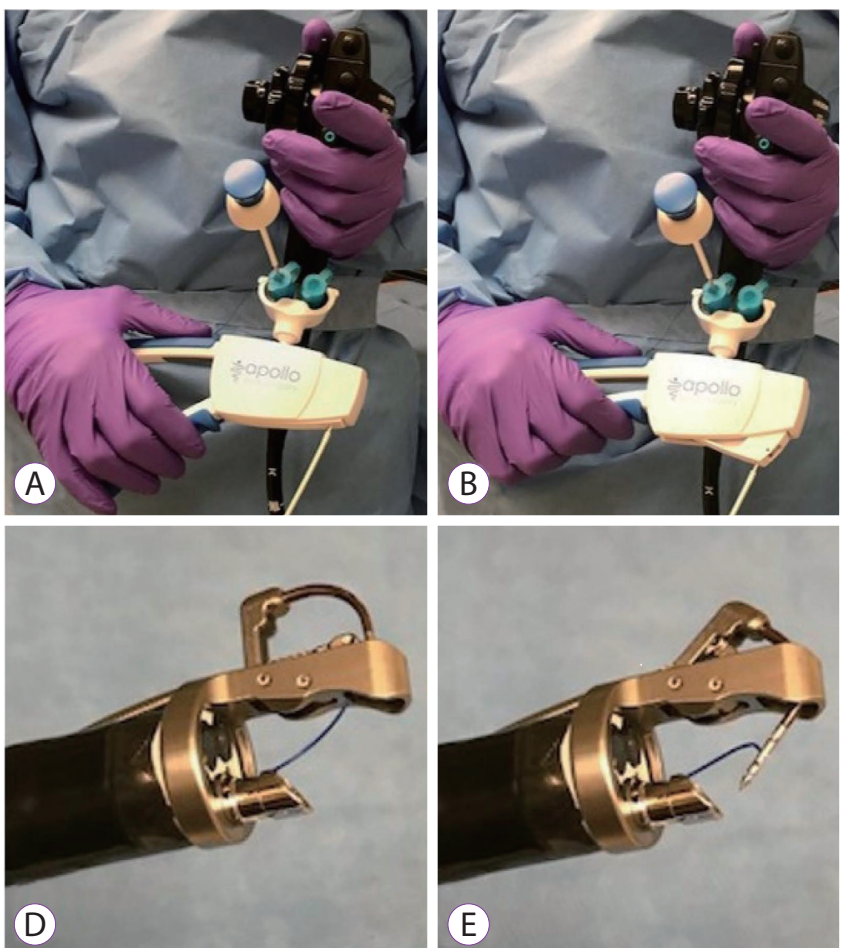
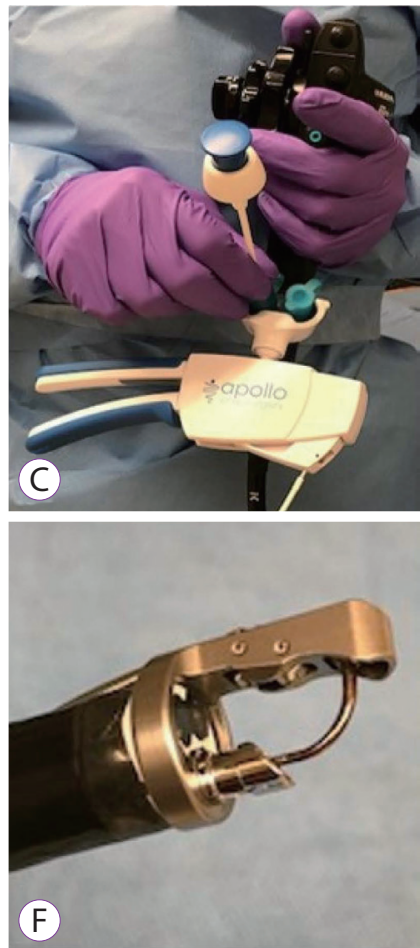

Fig. 2. Overstitch Endoscopic Suturing System. (A) The handle open corresponds to (D). (B) The handle being closed corresponds to (E). (C) Fully closed handle with anchor exchange catheter (pick-up) being advanced to remove the needle from the curved suture arm. This corresponds to (F). (D) The needle and suture loaded on the curved suture arm in a fully open configuration. (E) The needle and suture on the curved suture arm as it would appear being advanced through tissue. (F) The curved suture arm in a closed configuration. 

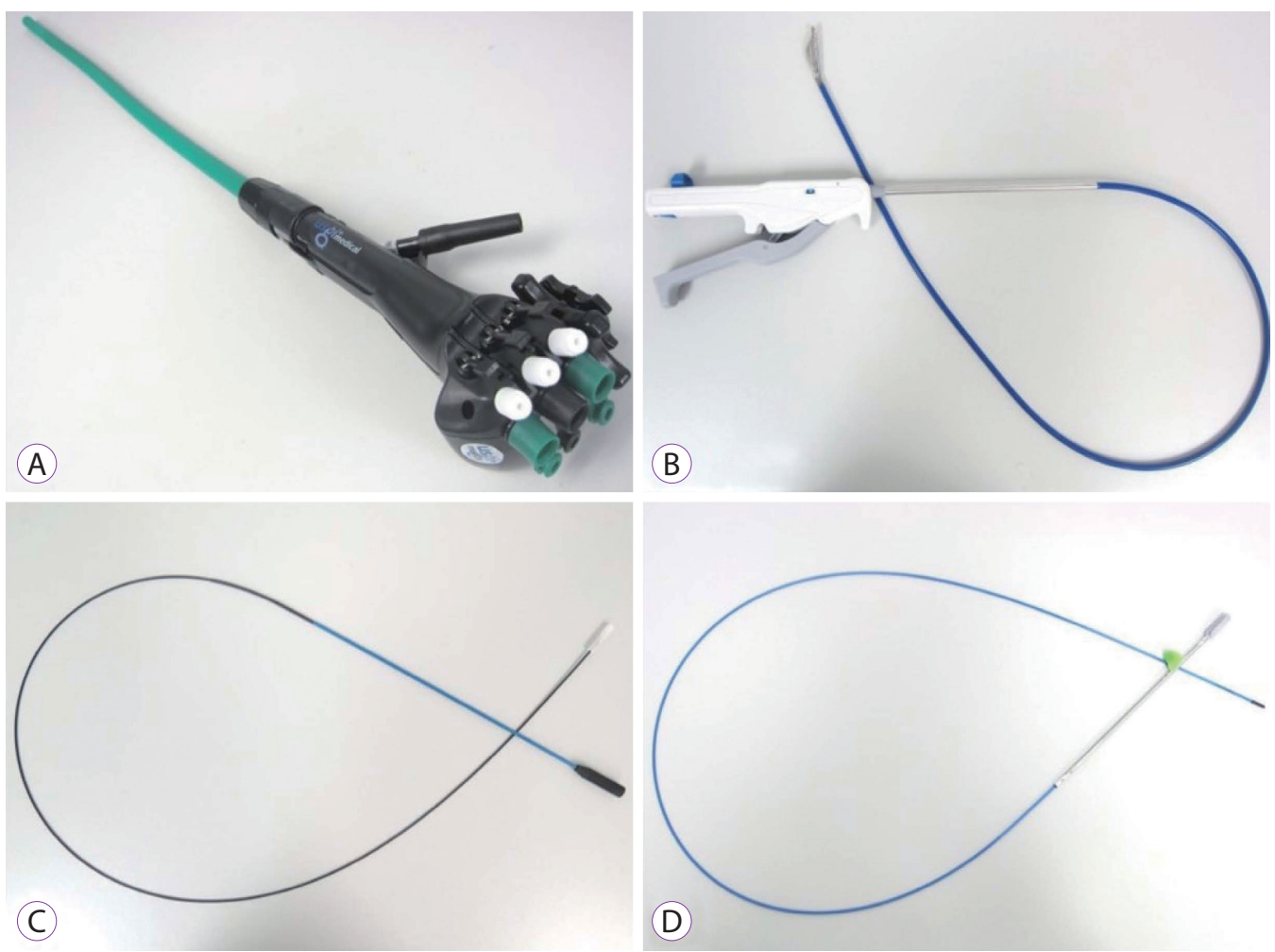

Fig. 3. The Incisionless Operating Platform System. (A) Transport. (B) G-Prox. (C) G-Cath. (D) G-Lix.

(Fig. 1F) ${ }^{20,21}$

This review article aims to summarize important elements of training in bariatric endoscopy. Specifically, goals, resources, and stages of training for both cognitive and technical elements are summarized. Lifestyle intervention, pharmacotherapy and management of complications of bariatric surgery are important. However, these are beyond the scope of this review.

\section{TRAINING GOALS}

\section{Establish a comprehensive understanding of obe- sity pathophysiology}

Trainees should understand current theories regarding how obesity occurs, including but not limited to genetic and epigenetic factors, metabolic abnormalities, hormonal imbalance and changes in the microbiome, motility and bile acid composition. Throughout training, trainees should learn to evaluate patients with obesity and its related comorbidities and post-bariatric surgical patients in a clinical setting. Additionally, they should learn to diagnose and treat obesity-related gastrointestinal conditions, including nonalcoholic fatty liver disease, gallstone disease, gastroesophageal reflux disease, Barrett's esophagus, and gastrointestinal malignancies. ${ }^{22,23}$

\section{Establish an understanding of the important ele- ments of an interdisciplinary obesity management team}

Trainees should learn about available treatment options for obesity, which include the various diet plans, physical activity, medical therapy, endoscopic therapy and surgery. In addition, an emphasis on an interdisciplinary team strategy is important. Rotation through different services above, or through an interdisciplinary bariatric center of excellence (if available), should be considered to emphasize the importance of integrative care.

\section{Establish an understanding of post-bariatric sur- gical anatomy}

Trainees should understand the anatomy of different types of bariatric surgery. ${ }^{24}$ Additionally, when the type of surgery is unknown, trainees should learn to identify the specific type based on endoscopic findings. Understanding possible complications of each bariatric surgery and how to medically and endoscopically manage those complications are also key.

\section{Establish technical proficiency in endoscopic bar- iatric and metabolic therapies}

Trainees should be able to identify the currently available EBMT devices and procedures, the patient population that 
the device is approved for, and possible adverse events. ${ }^{21,25,26}$ In general, trainees should start by learning level I procedures and then incorporate various level II procedures as progress dictates.

\section{Establish a curriculum consistent with the Amer- ican Boards of Obesity Medicine requirements for certification}

For a subset of trainees in the U.S., who plan on specializing in bariatrics, their goal of training should also include becoming board certified in Obesity Medicine. Specifically, trainees may become Board eligible if they have spent greater than 500 hours (approximately 2.5 full-time months) on clinical rotations managing patients with obesity and/or obesity-related conditions. ${ }^{27}$ There is also a Continuing Medical Education (CME)-based path for practicing clinicians including a minimum of $60 \mathrm{CME}$ credits with at least 30 credits being earned through attendance of group one meetings (Obesity Medicine Association Spring conference, Obesity Medicine Association Fall Conference, Columbia University/Weill Cornell Obesity Course, Harvard Blackburn Course in Obesity Medicine and Obesity Week) and the remainder of the 30 credits through participation in group two activities, including home-based CME activities and live meetings where obesity is the focus of interest.

Composition of the above training goals will differ based on level of training and specific interest. For advanced fellows seeking to perform full range of primary therapies, goals 1 to 5 are all important. For general GI fellows, goals 1 to 2 with a primary focus on general clinical exposure to the patient population and bariatric surgical complications may be sufficient. Lastly, for practicing clinicians, the goals will vary, but should include goals 1 and 2 with goals 3 to 5 being pursued as necessary.

\section{INFRASTRUCTURE AND PERSONNEL}

\section{Facilities}

Most bariatric procedures are considered high risk and should be performed in a hospital setting. However, for some of the level I procedures, they may take place in an ambulatory surgery center after thorough review of cardiopulmonary and procedure risks. The facility should be equipped to accommodate bariatric patients in a sensitive manner. These include having larger armless chairs, stretchers with appropriate width, obesity scales, larger blood pressure cuffs readily. Additionally, the facility should be part of the bariatric center of excellence, where patients may receive care from the medical bariatrician, bariatric surgeon, nutritionist, psychologist and lifestyle coach. New web-based technology or an outside consultant may be considered if ancillary nutrition services are not available at an institution.

\section{Educators}

Educators should have expertise in both medical bariatrics and endoscopic bariatrics. If a GI division does not have a bariatric specialist, a multidivisional team of faculty should be formed to provide the training. These specialties should include nutrition, exercise physiology, lifestyle coaching, obesity medicine, endocrinology, psychology as well as bariatric surgery. For institutions that this multidisciplinary group of faculty is not available, didactic and hands-on courses hosted by different universities and professional societies (see the additional training resource section) may provide an alternative solution.

\section{Trainees}

Currently, the level of endoscopic experience required prior to undergoing training in bariatric endoscopy is unclear. Trainees should be at least comfortable with standard upper endoscopy and basic therapeutic techniques, such as hemostasis. Prior experience in interventional endoscopy, such as endoscopic ultrasound or endoscopic retrograde cholangiopancreatography, is not mandatory. Bariatric endoscopy training may be pursued during the standard 3-year GI fellowship (if faculty with bariatric expertise are available at the trainee's institution) or as an additional 4th year fellowship after the standard 3-year GI fellowship (if faculty with bariatric expertise are unavailable at the trainee's institution).

\section{TRAINING PROCESS}

Training in bariatric endoscopy consists of cognitive and technical training. The cognitive aspects of bariatric endoscopy include understanding the pathophysiology of obesity, different management options for obesity including lifestyle therapy, medications, bariatric endoscopy and bariatric surgery) and in which patient population and when bariatric endoscopy should be considered. ${ }^{28-32}$ A clear understanding of EBMT devices and procedures including their mechanisms of action, efficacy and risk profile are crucial. Additionally, an understanding of the anatomy and physiology of each bariatric surgery is an important step to effectively manage patients with surgical complications.

\section{Patient selection}

Selecting an appropriate therapy for each patient is important to optimize clinical outcomes. Despite a shared goal of 
inducing weight loss, each EBMT device has different mechanisms of actions and therefore may be appropriate for different patient sub-populations.

IGBs are approved for the body mass index (BMI) range of 30 to $40 \mathrm{~kg} / \mathrm{m}^{2},{ }^{10-12}$ while aspiration therapy is approved for patients with a BMI between 35 and $55 \mathrm{~kg} / \mathrm{m}^{2} .{ }^{20}$ In general, ESG and POSE have been used for patients with a BMI of greater than $30 \mathrm{~kg} / \mathrm{m}^{2}$ who failed noninvasive weight loss measures, or those with a BMI of greater than $40 \mathrm{~kg} / \mathrm{m}^{2}$ who were not considered surgical candidates or refused surgery. ${ }^{14,17-19}$ On average, IGBs are associated with approximately $7 \%-10 \%$ TWL, ${ }^{10-12}$ while ESG and POSE have been reported to result in approximately $20 \%$ TWL. ${ }^{14,17-19}$ Patients who undergo aspiration therapy can experience about 12\% TWL per year over the first few years of therapy. ${ }^{20}$

In making decisions regarding which EBMT to provide, initial BMI, ideal body weight, comorbidities, financial status and risk aversion should be considered. In addition, for patients who consider aspiration therapy, discussion regarding how to fit aspiration into their daily life should take place prior to the procedure. Patients who may benefit more from bariatric surgery should be encouraged to revisit the option.

\section{Preprocedural assessment}

A comprehensive understanding of pre-procedural assessment is crucial. Specifically, targeted past medical and surgical history, current medications, history of prior weight loss attempts and smoking history should be obtained. Consultation with a cardiology or neurologist should be pursued for patients who are on an anticoagulation or an antiplatelet to determine if the medication can be held safely peri-procedurally. If not, the procedures should be postponed until the antiplatelet or anticoagulation can be safely held.

Trainees should understand the pre-operative instructions for each procedure. These instructions may include the requirement of proton pump inhibitor (PPI) or anti-emetic administration prior to the procedure. Some anti-emetics, such as Apprepitant (Emend), may require prior authorization from the insurance company, which should be arranged in advance. In addition, for some procedures, such as argon plasma coagulation (APC) and TORe, an arrangement for a liquid or crushable form of the patient's medications should be done prior to the procedure in collaboration with the specialists who prescribe the medications and the pharmacist.

Prior to the procedure, trainees should understand the components of each device and how they work. Detailed procedural steps should be reviewed with the mentor, especially during the initial phase of training, and with assisting nurse or technician.

\section{Procedural considerations and techniques}

Similar to other standard endoscopic training, training in bariatric endoscopy should gradually progress from level I (less complex) to level II (more complex) (level II) procedures. Trainees should start by learning to assist prior to performing the procedure. Subsequently, they may take on simpler steps with less risk involved prior to progressing to more technically challenging and invasive steps prior to becoming independent. The learning curve and learning style for each trainee may vary. Therefore, specific feedback from the mentor during and after each procedure especially during the initial phase of training is essential. This section summarizes key training steps for the primary EBMTs and endoscopic procedures for the treatment of weight regain following bariatric surgery. Additionally, it is important to learn not only how to perform these procedures safely but also how to troubleshoot device failures and deal with complications that may arise during and after the procedures.

\section{Level 1 procedures}

\section{Intragastric balloons}

Different IGBs are associated with distinct steps of balloon placement and removal. Prior to the procedure, these steps should be reviewed using a combination of the company's instructional video, live case demonstration and practicing and rehearsing with the mentor. Steps that are considered less complex where trainees may start assisting include filling the balloon with normal saline with or without methylene blue. Subsequently, trainees may start passing the balloon catheter through the upper esophageal sphincter. Attention should be paid to avoid significant torqueing or harsh pressure to prevent upper esophageal tear. During IGB removal, trainees may start by learning to perform needle puncture to deflate the balloon. Subsequently, balloon removal may be pursued with careful attention paid to avoid harsh force if there is resistance at the lower esophageal sphincter to prevent trauma and tear. Instead, the scope along with the snare and deflated balloon should be re-aligned before removal is re-attempted. Trainees should also learn to identify and manage potential adverse events following IGB placement and removal, which include nausea, vomiting, dehydration, abdominal spasm, balloon leak, balloon hyperinflation, gastric perforation, small bowel obstruction and rarely pancreatitis. ${ }^{10-12}$

\section{Aspiration therapy}

The AspireAssist system consists of an A-tube, which is a large percutaneous endoscopic gastrostomy (PEG) tube with a long fenestrated tube inside the stomach. The device allows removal of approximately $30 \%$ of ingested calories at 20 to 
30 minutes after a meal. Trainees who are comfortable with standard PEG placement may start by performing the percutaneous steps of the A-tube placement using the standard pull technique. Given the large diameter of the A-tube, a small cut on the skin may be required. For trainees who are not yet comfortable with PEG placement, he or she may start by assisting with the endoscopy steps, while learning the details of the percutaneous steps. Seven to fourteen days after A-tube placement, the tube is cut to a skin level and the port is connected. Trainees should have a comprehensive understanding of how the device works prior to instructing patients on how to perform aspiration. Similar to other EBMTs, trainees should learn to identify and manage potential adverse events following aspiration therapy, which include granulation tissue (treated with silver nitrate), irritation, infection, abdominal pain, buried bumper and electrolyte disturbances. ${ }^{20}$

\section{Level II procedures}

\section{Transoral outlet reduction for weight regain}

The Overstitch Endoscopic Suturing System (Apollo Endosurgery) consists of a handle, which is attached to a double channel endoscope, and a curved suture arm and an anchor exchange, which extends from each of the working channels (Fig. 2). The trainee should first familiarize themselves with the device and practicing with a simulator prior to the initial human case. Key steps during TORe should be frequently reviewed which include (1) loading the needle and suture onto the curved suture arm (2) repositioning the scope in order to appropriately advance the needle and suture through the tissue in a perpendicular orientation to ensure deep full-thickness tissue acquisition (3) repeating steps 1 and 2 for a minimum of 12 stitches until the entire purse string is created around the gastrojejunal anastomosis (GJA) (4) dropping the needle which now serves as a tissue anchor (5) cinching around the inflated $8 \mathrm{~mm}$ hydrostatic balloon to ensure a proper final GJA size. TORe using the interrupted suture pattern is less technically demanding and may be useful early in the learning curve. ${ }^{13,15}$ However, more recent studies showed that TORe using a purse string suture technique appears to be able to induce more weight loss compared to interrupted suture technique. ${ }^{28-30}$ Therefore, the trainee should learn to become comfortable at performing both techniques. For purse string TORe, the less involved steps include tissue preparation with APC. Once the trainee are comfortable with this step, they may start suturing. It is important to note that the first stitch is usually placed at a 5 oclock position by rotating the device and body. In general, the last several stitches are the easiest to perform and may be a good place to start suturing for novices. Therefore, the trainee will naturally progress from being able to place a few stitches to being able to complete the entire purse string independently. Post-procedural care includes opened PPI, i.e., opening the PPI capsule and mixing the medication in apple sauce to ensure adequate absorption given rapid emptying following Roux-en-Y gastric bypass, soluble Carafate, smoking and nonsteroidal anti-inflammatory drugs avoidance and liquid diet for 6 weeks. Possible adverse events include nausea, vomiting, severe constipation, gastrointestinal bleeding and GJA stricture. ${ }^{29}$ It is important for the trainee to learn to recognize these possible adverse events early and manage them appropriately.

\section{Restorative obesity surgery endoluminal for weight regain}

The IOP system (USGI Medical) consists of four components: the transport-R, G-lix, G-prox and G-cath (Fig. 3). The transport is a 54-French flexible scope with a control handle and four working channels, one of which is for an insertion of an ultraslim endoscope for visualization. The G-lix is used for tissue grasping prior to tissue approximation by the G-prox and tissue plication by the G-cath. A comprehensive understanding of these device components and key steps involved in placing a plication is essential. Additionally, patient selection for ROSE is of importance to technical and clinical success. Typically, this includes patients with a dilated GJA of at least $10 \mathrm{~mm}$ and a long pouch of at least $4 \mathrm{~cm}$. During ROSE training, trainees may start by providing visualization and stabilization of the platform Plications are typically placed distally to proximally, i.e., from around the GJA to the distal pouch to the proximal pouch. The least technically challenging plication location is usually in the distal pouch, followed by the proximal pouch and around the GJA, respectively. Post-procedurally, patients should be given opened PPI, liquid or crushable medications and liquid diet for 6 weeks. Possible adverse events include nausea, vomiting, severe constipation, gastrointestinal bleeding and GJA stricture.

\section{Endoscopic sutured gastroplasty}

If possible, we recommend that a trainee become familiar with TORe prior to advancing to ESG. This is in large part due to the risk profile and duration of ESG being much longer. During ESG, multiple sets of running suture are placed from the distal body to the proximal body. For each set of running suture, at least six stitches are placed in a triangular or U-shaped fashion at the anterior wall, greater curvature and posterior wall. Trainees may start by learning to load the device with the suture and assisting with the cinching process. Subsequently, they may progress to start placing stitches. In general, placing stitches in the distal body is less technically challenging than those in the proximal body and should be a good starting location for the trainee. Furthermore, initial 
stitches are easier to place in any configuration due to lower tension. It is important to note that a helix is often needed to ensure appropriate depth of each tissue bite and proper helix use must be taught and assessed. Post-procedural care includes antibiotics for a few days, PPI for 6 weeks, and liquid diet for 2 weeks followed by pureed diet for 2 weeks then regular diet. Possible adverse events that the trainee should learn to manage include abdominal pain, nausea, vomiting, constipation, leak and intraperitoneal bleeding. ${ }^{14,17}$

\section{Primary obesity surgery endoluminal}

The IOP system that is used for POSE is similar to that that is used for ROSE except that the transport has more flexibility at performing retroflexion and the G-prox is longer in length (33 mm instead of $18 \mathrm{~mm}$ ). The POSE techniques may be divided into the traditional POSE and distal POSE techniques. Both require similar skills but differ in the location of plication placement. Specifically, in the traditional POSE, several plications are placed in the fundus (in a retroflexed fashion) and a few plications in the distal body. In contrast, distal POSE places all of the plications in the gastric body with the goal of reducing the gastric volume circumferentially and longitudinally, indirectly reducing fundic capacity. Similar to ROSE, the trainee should first become familiar with the device and the steps involved in deploying each plication. Subsequently, understanding the plication pattern is essential prior to performing the procedure. In general, distal plications are technically less challenging than those in the proximal location. Careful attention should be paid at training proper use of the G-lix and G-prox devices in order to maximize the depth of each plication. After the procedure, patients are prescribed antibiotics for a few days, PPI for 6 weeks, and similar diet plan as the ESG procedure. Possible adverse events include abdominal pain, nausea, vomiting, leak and intraperitoneal bleeding. ${ }^{18,19}$

\section{Postprocedural considerations}

Trainees should understand specific post-procedural diet, medications and activity limitations following each EBMT procedure. Communications of procedural findings and follow-up plans should be discussed with the patient and family. For patients who are admitted, instructions should be discussed with the inpatient team in a timely manner. In addition, the importance of adjunctive lifestyle therapy should be emphasized following every bariatric procedure.

\section{ASSESSMENT OF TRAINING}

Currently, there are no objective assessment tools to determine competency in bariatric endoscopy. A previous ques- tionnaire study surveying 10 experts in the field of bariatric endoscopy revealed that a minimum number of 15 and 22 TORe and primary bariatric endoscopic suturing cases were required for the trainee to become independent. ${ }^{31}$ These numbers are likely to be higher for more complex techniques, such as pursestring suturing TORe. In addition, these numbers were based primarily on the experience of training with experts in the field. Generalizability of this data therefore remains to be validated. In general, frequent feedback after clinic visits and endoscopic cases should be provided to the trainee. Furthermore for trainees who plan on specializing in the bariatric field, obtaining the American Board of Obesity Medicine certification should be highly encouraged.

\section{SIMULATORS}

Simulator training for bariatric endoscopy is an emerging field. Traditionally, trainees may start by learning the device and simple steps using ex-vivo animal models. These models are usually available at most hands-on sessions. Alternatively, a mechanical endoscopic suturing simulator may be used (Fig. 4). ${ }^{32}$ This simulator allows trainees to familiarize themselves with the endoscopic suturing device and to learn stitch placement to perform pursestring TORe. Furthermore, company and device specific models are becoming more available and trainees are encouraged to seek these training opportunities.

\section{ADDITIONAL TRAINING RESOURCES}

\section{Clinical guidelines}

White Paper AGA: POWER - Practice Guide on Obesity and Weight Management, Education, and Resources. ${ }^{33}$

AHA/ACC/TOS guideline for the management of overweight and obesity in adults: a report of the American College of Cardiology/American Heart Association Task Force on Practice Guidelines and The Obesity Society. ${ }^{2}$

ASGE Bariatric Endoscopy Task Force systematic review and meta-analysis assessing the ASGE PIVI thresholds for adopting endoscopic bariatric therapies. ${ }^{34}$

Advances in Obesity: Causes, consequences, and Therapy. ${ }^{35}$

\section{Videos}

The ASGE Video Tip of the Week: The Bariatric Series. https://www.asge.org/search-results?TypeFacet=VideoTips\&Categories=357e2164-1d27-6839-97eb-ff000074820c.

The ASGE Endoscopic Learning Library: The Bariatric DVD Set. 

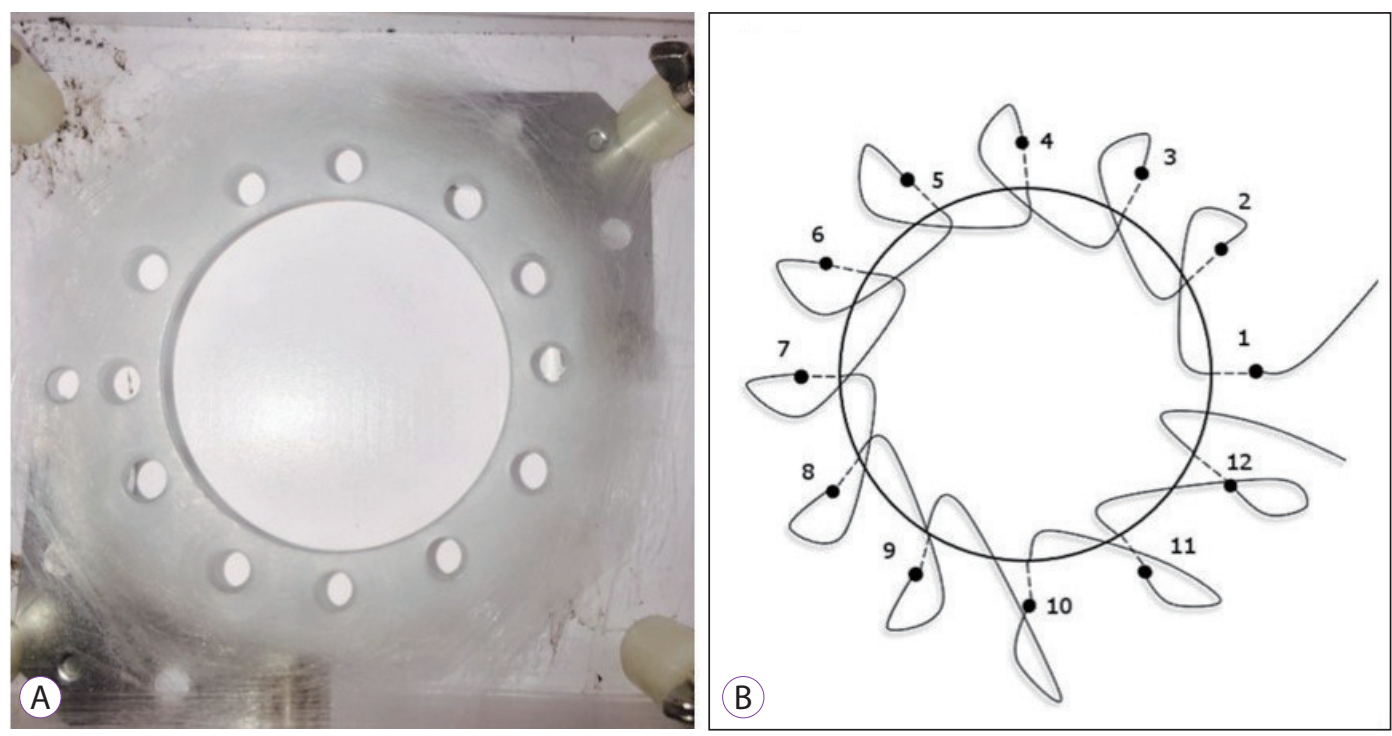

Fig. 4. An Endoscopic Suturing Simulator.

\section{Books}

Akabas SR, Lederman SA, Moore BJ. Textbook of obesity: biological, psychological and cultural influences. Hoboken (NJ): Wiley-Blackwell; 2012.

Steelman GM, Westman EC. Obesity: evaluation and treatment essentials. 2nd ed. Boca Raton (FL): CRC Press; 2016.

Youdim A. The clinician's guide to the treatment of obesity. Boston (MA); Springer; 2015.

Thompson CC. Bariatric endoscopy: indispensible tool for the gastroenterologist or surgical endoscopist. New York (NY): Springer; 2013.

\section{Courses}

The Blackburne Course in Obesity Medicine. Held annually in Boston, MA, USA.

The Obesity Society Review Course for the American Board of Obesity Medicine Exam. Held annually at the Obesity Week conference.

The American Bariatric Endoscopy (ABE) Bariatric Course. Held annually at the ASGE IT\&T center in Chicago, IL, USA.

The Flexible Endoscopic Surgery and Bariatric Endoscopy Course. Held annually in Miami, FL, USA.

The Madrid International Bariatric Endoscopy Meeting (MIBE) Course. Held annually in Madrid, Spain.

\section{CONCLUSIONS}

Training in EBMTs requires a multidisciplinary approach. Understanding the pathophysiology of obesity and the subtleties of medical and endoscopic therapies, and when bariatric endoscopy should or should not be offered are important. Once trainees are comfortable with the cognitive aspects of bariatric endoscopy, training of the technical aspects may advance from less complex to more complex procedures. As this field continues to evolve, structured curricula will provide more complete guidance regarding training and credentialing.

\section{Conflicts of Interest}

Pichamol Jirapinyo has no conflicts of interest. Christopher C. Thompson has received consulting fees from Boston Scientific, Covidien, USGI Medical, Valentx, and Apollo Endosurgery, has served as an advisory board member for USGI Medical, has served as an advisory board member for Fractyl, has received research/grant support from USGI Medical and Apollo Endosurgery, has received laboratory supplies and equipment from Olympus, has received trial funding from Aspire bariatrics and Spatz, has served as a consultant for Olympus and Fractyl, has served as an expert reviewer for GI Dynamics, and has an ownership interest in GI Windows.

\section{REFERENCES}

1. World Health Organization. Obesity and overweight [Internet]. Geneva: WHO; c2018 [updated $2018 \mathrm{Feb}$ 16; cited 2018 Jun 29]. Available from: http://www.who.int/news-room/fact-sheets/detail/obesity-and-overweight.

2. Jensen MD, Ryan DH, Apovian CM, et al. 2013 AHA/ACC/TOS guideline for the management of overweight and obesity in adults: a report of the American College of Cardiology/American Heart Association Task Force on Practice Guidelines and The Obesity Society. J Am Coll Cardiol 2014;63(25 Pt B):2985-3023.

3. Look AHEAD Research Group. Eight-year weight losses with an intensive lifestyle intervention: the look AHEAD study. Obesity (Silver Spring) 2014;22:5-13 .

4. Igel LI, Kumar RB, Saunders KH, Aronne LJ. Practical use of pharmacotherapy for obesity. Gastroenterology 2017;152:1765-1779.

5. Corcelles R, Boules M, Froylich D, et al. Total weight loss as the out- 
come measure of choice after Roux-en-Y gastric bypass. Obes Surg 2016;26:1794-1798.

6. Ponce J, Nguyen NT, Hutter M, Sudan R, Morton JM. American Society for Metabolic and Bariatric Surgery estimation of bariatric surgery procedures in the United States, 2011-2014. Surg Obes Relat Dis 2015;11:1199-1200.

7. Abu Dayyeh BK, Eaton LL, Woodman G, et al. A randomized, multi-center study to evaluate the safety and effectiveness of an intragastric balloon as an adjunct to a behavioral modification program, in comparison with a behavioral modification program alone in the weight management of obese subjects. Gastrointest Endosc 2015;81(5 Suppl):AB147.

8. Ponce J, Woodman G, Swain J, et al. The REDUCE pivotal trial: a prospective, randomized controlled pivotal trial of a dual intragastric balloon for the treatment of obesity. Surg Obes Relat Dis 2015;11:874-881.

9. Sullivan S, Swain JM, Woodman G, et al. The obalon swallowable 6-month balloon system is more effective than moderate intensity lifestyle therapy alone: results from a 6- month randomized sham controlled trial. Gastroenterology 2016;150(4 Suppl):S1267.

10. US Food and Drug Administration. Summary of safety and effectiveness data (SSED): ORBERA ${ }^{\mathrm{Tx}}$ intragastric balloon system [Internet] Silver Spring (MD): FDA; c2015 [updated 2015 Aug 5; cited 2018 Sep 19]. Available from: https://www.accessdata.fda.gov/cdrh_docs/pdf14/ P140008b.pdf.

11. US Food and Drug Administration. Summary of safety and effectiveness data (SSED): obalon balloon system [Internet]. Silver Spring (MD): FDA; c2016 [updated 2016 Sep 8; cited 2018 Sep 19]. Available from: https://www.accessdata.fda.gov/cdrh_docs/pdf16/P160001b.pdf.

12. US Food and Drug Administration. Summary of safety and effectiveness data (SSED): ReShape ${ }^{\mathrm{Tx}}$ integrated dual balloon system [Internet] Silver Spring (MD): FDA; c2015 [updated 2015 Jul 28; cited 2018 Sep 19]. Available from: https://www.accessdata.fda.gov/cdrh_docs/pdf14/ P140012b.pdf.

13. Jirapinyo P, Slattery J, Ryan MB, Abu Dayyeh BK, Lautz DB, Thompson CC. Evaluation of an endoscopic suturing device for transoral outlet reduction in patients with weight regain following Roux-en-Y gastric bypass. Endoscopy 2013;45:532-536.

14. Sharaiha RZ, Kedia P, Kumta N, et al. Initial experience with endoscopic sleeve gastroplasty: technical success and reproducibility in the bariatric population. Endoscopy 2015;47:164-166.

15. Kumar N, Thompson CC. Transoral outlet reduction for weight regain after gastric bypass: long-term follow-up. Gastrointest Endosc 2016;83:776-779.

16. Lopez-Nava G, Galvao M, Bautista-Castaño I, Fernandez-Corbelle JP, Trell M. Endoscopic sleeve gastroplasty with 1-year follow-up: factors predictive of success. Endosc Int Open 2016;4:E222-E227.

17. Lopez-Nava G, Sharaiha RZ, Vargas EJ, et al. Endoscopic sleeve gastroplasty for obesity: a multicenter study of 248 patients with 24 months follow-up. Obes Surg 2017;27:2649-2655.

18. Miller K, Turró R, Greve JW, Bakker CM, Buchwald JN, Espinós JC. MILEPOST multicenter randomized controlled trial: 12-month weight loss and satiety outcomes after pose (SM) vs. medical therapy. Obes
Surg 2017;27:310-322

19. López-Nava G, Bautista-Castaño I, Jimenez A, de Grado T, Fernandez-Corbelle JP. The primary obesity surgery endolumenal (POSE) procedure: one-year patient weight loss and safety outcomes. Surg Obes Relat Dis 2015;11:861-865.

20. Thompson CC, Abu Dayyeh BK, Kushner R, et al. Percutaneous gastrostomy device for the treatment of class II and class III obesity: results of a randomized controlled trial. Am J Gastroenterol 2017;112:447-457.

21. Jirapinyo P, Thompson CC. Endoscopic bariatric and metabolic therapies: surgical analogues and mechanisms of action. Clin Gastroenterol Hepatol 2017;15:619-630.

22. Foxx-Orenstein AE. Gastrointestinal symptoms and diseases related to obesity: an overview. Gastroenterol Clin North Am 2010;39:23-37.

23. Camilleri M, Malhi H, Acosta A. Gastrointestinal complications of obesity. Gastroenterology 2017;152:1656-1670.

24. Jirapinyo P, Kumar N, Thompson CC. Patients with Roux-en-Y gastric bypass require increased sedation during upper endoscopy. Clin Gastroenterol Hepatol 2015;13:1432-1436.

25. ASGE/ASMBS Task Force on Endoscopic Bariatric Therapy. A pathway to endoscopic bariatric therapies. Surg Obes Relat Dis 2011;7:672-682.

26. Sullivan S, Edmundowicz SA, Thompson CC. Endoscopic bariatric and metabolic therapies: new and emerging technologies. Gastroenterology 2017;152:1791-1801.

27. Kushner RF, Brittan D, Cleek J, et al. The American board of obesity medicine: five-year report. Obesity (Silver Spring) 2017;25:982-984.

28. Patel LY, Lapin B, Brown CS, et al. Outcomes following 50 consecutive endoscopic gastrojejunal revisions for weight gain following Roux-en-Y gastric bypass: a comparison of endoscopic suturing techniques for stoma reduction. Surg Endosc 2017;31:2667-2677.

29. Jirapinyo P, Kröner PT, Thompson CC. Purse-string transoral outlet reduction (TORe) is effective at inducing weight loss and improvement in metabolic comorbidities after Roux-en-Y gastric bypass. Endoscopy 2018;50:371-377.

30. Schulman AR, Kumar N, Thompson CC. Transoral outlet reduction: a comparison of purse-string with interrupted stitch technique. Gastrointest Endosc 2018;87:1222-1228.

31. Jirapinyo P, Kumar N, Thompson CC. Endoscopic suturing: indications, procedural complexity and learning curve. Gastrointest Endosc 2016;83(5 Suppl):AB494-AB495.

32. Skinner MJ, Aihara H, Jirapinyo P, Thompson CC. Development and initial validation of a fully synthetic and reusable endoscopic suturing simulator. Gastrointest Endosc 2017;85(5 Suppl):AB502-AB503.

33. Acosta A, Streett S, Kroh MD, et al. White paper AGA: POWER - practice guide on obesity and weight management, education, and resources. Clin Gastroenterol Hepatol 2017;15:631-649.e10.

34. ASGE Bariatric Endoscopy Task Force and ASGE Technology Committee, Abu Dayyeh BK, Kumar N, et al. ASGE bariatric endoscopy task force systematic review and meta-analysis assessing the ASGE PIVI thresholds for adopting endoscopic bariatric therapies. Gastrointest Endosc 2015;82:425-438.e5.

35. Berthoud HR, Klein S. Advances in obesity: causes, consequences, and therapy. Gastroenterology 2017;152:1635-1637. 